\title{
Vandetanib inhibits both VEGFR-2 and EGFR signalling at clinically relevant drug levels in preclinical models of human cancer
}

\author{
SANDRA R. BRAVE ${ }^{1}$, RAJESH ODEDRA ${ }^{1}$, NEIL H. JAMES ${ }^{1}$, NEIL R. SMITH ${ }^{1}$, GAYLE B. MARSHALL ${ }^{1}$, \\ KERRY L. ACHESON ${ }^{1}$, DAWN BAKER ${ }^{1}$, ZOE HOWARD ${ }^{1}$, LYNSAY JACKSON ${ }^{1}$, \\ KIRSTY RATCLIFFE $^{1}$, ANNA WAINWRIGHT ${ }^{1}$, SUSAN C. LOVICK ${ }^{1}$, D. MARK HICKINSON ${ }^{1}$, \\ ROBERT W. WILKINSON ${ }^{1}$, SIMON T. BARRY ${ }^{1}$, GEORGINA SPEAKE ${ }^{1}$ and ANDERSON J. RYAN ${ }^{1,2}$ \\ ${ }^{1}$ Cancer Bioscience, AstraZeneca Pharmaceuticals, Alderley Park, Macclesfield, \\ Cheshire SK10 4TG; ${ }^{2}$ Gray Institute for Radiation Oncology \& Biology, University of Oxford, \\ Old Road Campus Research Building, Roosevelt Drive, Headington, Oxford OX3 7DQ, UK
}

Received January 17, 2011; Accepted February 18, 2011

DOI: $10.3892 /$ ijo.2011.1022

\begin{abstract}
Vandetanib is a multi-targeted receptor tyrosine kinase inhibitor that is in clinical development for the treatment of solid tumours. This preclinical study examined the inhibition of two key signalling pathways (VEGFR-2, EGFR) at drug concentrations similar to those achieved in the clinic, and their contribution to direct and indirect antitumour effects of vandetanib. For in vitro studies, receptor phosphorylation was assessed by Western blotting and ELISA, cell proliferation was assessed using a cell viability endpoint, and effects on cell cycle determined using flow cytometry. For in vivo studies, Western blotting, ELISA and immunohistochemistry (IHC) were used to assess receptor phosphorylation. Cell culture experiments demonstrated that anti-proliferative effects of vandetanib resulted from inhibition of either EGFR or VEGFR-2 signalling in endothelial cells, but were associated with inhibition of EGFR signalling in tumour cells. Vandetanib inhibited both EGFR and VEGFR-2 signalling in normal lung tissue and in tumour xenografts. In a lung cancer model expressing an activating EGFR mutation, the activity of vandetanib was similar to that of a highly selective EGFR inhibitor (gefitinib), and markedly greater than that of a highly selective VEGFR inhibitor (vatalanib). These data suggest that at the plasma exposures achieved in the clinic, vandetanib will significantly inhibit both VEGFR-2 and EGFR signalling, and that both inhibition of angiogenesis and direct inhibition of tumour cell growth can contribute to treatment response.
\end{abstract}

Correspondence to: Dr Anderson J. Ryan, Present address: Gray Institute for Radiation Oncology \& Biology, University of Oxford, Old Road Campus Research Building, Roosevelt Drive, Oxford OX3 7DQ, UK

E-mail: anderson.ryan@rob.ox.ac.uk

Key words: vandetanib, EGFR, VEGFR-2, lung cancer, xenografts, endothelial cells

\section{Introduction}

Vandetanib (ZD6474) was identified as a potent nanomolar inhibitor of vascular endothelial growth factor (VEGF) signalling with pharmacokinetic properties compatible with chronic, once daily, oral dosing $(1,2)$. Although the compound was also known to be a sub-micromolar inhibitor of epidermal growth factor receptor (EGFR) tyrosine kinase, the early preclinical evidence suggested that this activity is not essential for antitumour effects in vivo since vandetanib demonstrated significant inhibitory activity against a histologically diverse panel of human tumour xenografts, including models insensitive to gefitinib, a highly selective EGFR tyrosine kinase inhibitor (TKI) $(1,3)$. In the clinic, vandetanib has shown activity in non-small cell lung cancer (NSCLC) both as monotherapy and in combination with chemotherapy (4-6). Vandetanib monotherapy has an acceptable safety and tolerability profile at doses of up to $300 \mathrm{mg} / \mathrm{day}$, where the steady-state mean plasma drug concentration is approximately $1000 \mathrm{ng} / \mathrm{ml}(2.2 \mu \mathrm{M}$; range 1.6-6.3 $\mu \mathrm{M})$, and where adverse events included hypertension, rash and diarrhoea $(7,8)$ which is consistent with pharmacological inhibition of both VEGFR receptor-2 (VEGFR-2) and EGFR in normal tissues.

Although there has been some success in identifying biomarkers of differential benefit to selective EGFR signalling inhibitors (9), no predictive biomarkers have yet been identified for VEGF-signalling inhibitors (10). Identifying biomarkers of benefit from vandetanib, which has the potential to inhibit both VEGF and EGFR signalling in the clinic, has been hampered by insufficient data demonstrating how the agent is working in patients. Inhibition of VEGF and EGFR activity by vandetanib has been reported in small clinical studies, but with inconsistent results $(11,12)$. In light of the limited clinical data, the present work, a 'bedside-to-bench' translational study, was undertaken to better understand the potential mechanism of action of vandetanib in NSCLC and other solid tumours in the clinic, by utilising preclinical models as amenable systems 
to investigate vandetanib target inhibition at drug levels overlapping with those seen in the clinic.

\section{Materials and methods}

Animal studies. All in vivo experiments were conducted in full accordance with the UK Home Office Animal (Scientific Procedures) Act 1986. Mice were given food and water $a d$ libitum and all animal procedures were carried out under a project license issued by the home office (London, UK). Tumour studies were performed according to United Kingdom Coordinating Committee on Cancer Research guidelines.

Drugs and recombinant proteins. Vandetanib and gefitinib were synthesized as previously described $(13,14)$. Vatalanib was synthesized by AstraZeneca (Macclesfield, UK). Recombinant human VEGF-A V $_{165}$ was prepared as described previously $(1,15)$. Recombinant EGF was obtained from R\&D Systems (Abingdon, UK).

Tumour cell lines. NSCLC: Calu-3, NCI-H2085, NCI-H1793, NCI-H2126, NCI-H522, NCI-H1563, NCI-H647, NCI-H2342, NCI-H2122, NCI-H358, NCI-H322, A549, DMS 114, NCI-H23, NCI-H838, NCI-H2291, Calu-6, NCI-H1299, NCI-H1975 and NCI-H1437 were purchased from ATCC (Manassas, VA). PC-9 was obtained from Professor K. Nishio (National Cancer Center Hospital, Tokyo, Japan) and HX147 was obtained from Dr G.M. Duchesne via Peter Hepburn (ICRF, Sutton, UK).

Squamous cell carcinoma of the head and neck (SCCHN) and esophageal: CAL27, FaDu, SCC-25, Detroit562, SCC-4, SW579, RPMI2650, Hs840.T, KB, HEp-2, SCC-9 were purchased from ATCC. DOK, PE/CA-PJ49, PE/CA-PJ34 (clone C12), KYSE-30, PE/CA-PJ41 (clone D2), PE/CA-PJ15 and OE21 were purchased from ECACC (Porton Down, UK). HN5 was obtained from the Ludwig Institute for Cancer Research. KYSE-450 was purchased from DSMZ (Braunschweig, Germany).

Colorectal: SW48, SK-CO-1, HCT-15, SW948, HCT-116 were purchased from ATCC. LS180, LoVo, COLO 320DM, SW480, HT-29, HCT-8, COLO 205, SW620 and SW403 were purchased from ECACC. RKO was obtained from the University of Baltimore (Baltimore, MD) and BE was obtained from Dr Jane Plumb (Beatson Institute for Cancer Research, Glasgow, UK).

Inhibition of $p V E G F R-2$ and pEGFR in HUVECs. Human umbilical vein endothelial cells (HUVECs; PromoCell, Heidelberg, Germany) were cultured in MCDB 131 medium (Invitrogen Technologies, UK) containing 10\% fetal calf serum (FCS). Following incubation overnight in $2 \%$ FCS, HUVECs were incubated in serum-free MCDB 131 (Invitrogen Technologies) for $2.5 \mathrm{~h}$ after which cells were pre-treated with vandetanib for $90 \mathrm{~min}$ and VEGF-A $(50 \mathrm{ng} / \mathrm{ml})$ or epidermal growth factor (EGF; $1 \mu \mathrm{g} / \mathrm{ml}$ ) for the last $5 \mathrm{~min}$. Cells were lysed in the following buffer: $50 \mathrm{mM}$ Tris- $\mathrm{HCl}, \mathrm{pH} 7.6 ; 137 \mathrm{mM}$ $\mathrm{NaCl} ; 10 \%$ glycerol; $0.1 \%$ Igepal; $0.1 \%$ SDS; $50 \mathrm{mM} \mathrm{NaF}$; $1 \mathrm{mM} \mathrm{Na} \mathrm{VO}_{4}$ and cocktail protease inhibitor tablets (Roche Diagnostics Ltd., Lewes, UK). Protein concentration in the lysates was determined using a bicinchoninic acid (BCA) assay kit (Pierce, Rockford, IL). Western blotting was performed on whole cell lysates (50-75 $\mu \mathrm{g}$ of protein loaded per lane) using standard SDS-PAGE methods with detection by enhanced chemiluminescence. Total and phosphorylated proteins were measured using antibodies to total VEGFR-2 [tVEGFR-2; CST 2249; Cell Signaling Technology, Inc. (CST), Beverley, MA], and phosphorylated VEGFR-2 (pVEGFR-2; CST 2248, Y1175); total EGFR (CST 2232) and pEGFR (44-788, Y1068; Biosource, UK). Phosphorylation was quantified using the ChemiGenius imaging system for chemiluminescence (Syngene, Frederick, MD).

Tumour cell growth inhibition assay. NSCLC, SCCHN and colon cell lines were seeded in RPMI-1640 medium containing $10 \%$ FCS at a growth density allowing logarithmic growth during the assay. The cells were allowed to settle for $4 \mathrm{~h}$ before treatment with vandetanib or gefitinib. Following incubation for 72-96 h, viable cell number was assessed using an MTS (3-(4,5-dimethylthiazol-2-yl)-5-(3-carboxymethoxyphenyl)-2-(4-sulfophenyl)-2H-tetrazolium, inner salt) end-point according to the manufacturer's instructions (CellTiter 96 AQueous One Solution Cell Proliferation Assay, \#G1111; Promega, Madison, WI). The $\mathrm{IC}_{50}$ (the concentration of inhibition required to give $50 \%$ inhibition of growth) was calculated and analyzed using Origin software (OriginLab, Northampton, MA). The Pearson correlation coefficient (r) comparing mean $\mathrm{IC}_{50}$ values for vandetanib and gefitinib was calculated using Excel (Microsoft) and statistical significance was assessed by ANOVA.

Inhibition of pEGFR in human NSCLC cells. PC-9 and H1975 cell lines were seeded in RPMI-1640 medium containing 10\% fetal calf serum (FCS) before treatment with vandetanib or gefitinib (4 h). Cell lysates were prepared and protein assay and Western blotting were performed as described above. Total and phosphorylated proteins were measured using antibodies to EGFR (CST 2232; CST, Beverley, MA) and pEGFR (44-788, Y1068; Biosource, UK). Additionally ELISA kits were used according to manufacturer's instructions to measure pEGFR (DYC1905, R\&D Systems) in cell lysates.

Cell cycle analysis by flow cytometry. PC-9, H1975, OE21 and Hep-2 cell lines were seeded in RPMI 1640 medium containing $10 \%$ FCS. The cells were allowed to settle for $4 \mathrm{~h}$ before treatment with vandetanib or gefitinib for 24-72 h followed by fixation using $80 \%$ ethanol. Samples were stained for DNA content using $20 \mu \mathrm{g} / \mathrm{ml}$ propidium iodide (Cambridge Bioscience, Cambridge, UK). Cell cycle was assessed using a FACSCalibur flow cytometer and CellQuest software (BectonDickinson, Oxford, UK). Data was analysed and quantified using ModFit statistical software (VSH, Topsham, ME).

Apoptosis assay. PC-9, H1975, OE21 and HEp-2 cell lines were seeded and allowed to settle as previously described before treatment with vandetanib or gefitinib for 24-72 $\mathrm{h}$. The percentage of cells staining positive for annexin $\mathrm{V}$ (BectonDickinson) was assessed using PCA-96 base system and Nexin V software (Guava Technologies, Stamford, UK).

Lung and tumour pharmacodynamic assays. To assess effects of vandetanib on VEGFR-2 and EGFR phosphorylation in 
murine lung tissue and on EGFR phosphorylation in human tumour tissue, experiments were conducted on 8-12-week-old female nude mice housed in individually ventilated cages. LoVo human colorectal cancer cells were maintained in DMEM (Invitrogen) containing 10\% fetal bovine serum (FBS; Biochrom, Berlin, Germany) and $1 \%$ L-glutamine (Invitrogen) at $37^{\circ} \mathrm{C}$ in the presence of $7.5 \% \mathrm{CO}_{2}$. Xenografts were established by subcutaneous injection of $1 \times 10^{7}$ cells in $100 \mu \mathrm{l}$ of media containing 50\% Matrigel (Becton-Dickinson, UK) into the flank. Tumour volumes were assessed up to three times per week and calculated as described previously (15). When the mean tumour volume reached $0.5 \mathrm{~cm}^{3}$, mice were randomised into treatment groups to receive once-daily oral dosing with vandetanib (25 or $100 \mathrm{mg} / \mathrm{kg}$ ) or vehicle (1\% Tween-80) for 1-4 days. On each day of dosing, three animals from each treatment group were sacrificed $2 \mathrm{~h}$ post-dose. All mice received $20 \mu \mathrm{g}$ of VEGF-A (i.v., $30 \mu \mathrm{l}$ volume) $5 \mathrm{~min}$ before sacrifice. Plasma, lungs and tumours were collected from each animal and snap frozen in liquid nitrogen until analysis. Frozen mouse lungs and tumours were homogenised in ice-cold lysis buffer [20 mM Tris, pH 7.5; 137 mM NaCl; $10 \%$ glycerol; $1 \%$ NP40; $50 \mathrm{mM} \mathrm{NaF} ; 1 \mathrm{mM} \mathrm{Na} \mathrm{VO}_{4} ; 1$ protease inhibitor tablet $/ 25 \mathrm{ml}$ buffer (Boehringer Ingelheim Ltd., Bracknell, UK)], then treated with $0.1 \%$ SDS and cleared by centrifugation. Western blotting was performed on lung or tumour lysates $(250 \mu \mathrm{g}$ protein loaded per lane) using standard SDS-PAGE methods with detection by enhanced chemiluminescence as described earlier. Based on previous experience, chemiluminescence data were log-transformed prior to statistical analysis by one- or two-way ANOVA. Where ANOVA $\mathrm{P}<0.05$, pairwise analysis of vandetanib treatment groups versus control was subsequently performed using the Bonferroni method to correct for multiple testing. Total and phosphorylated proteins were measured using antibodies to VEGFR-2 (CST 2249) and pVEGFR-2 (CST 2248, Y1175); EGFR (CST 2232) and pEGFR (44-788, Y1068; Biosource). Frozen plasma was analyzed for total vandetanib drug levels as previously described (8).

Immunohistochemistry for VEGFR-2 and microvessel density $(M V D)$. To determine the effects of vandetanib on VEGFR-2 phosphorylation and MVD in murine lung tumours, immunohistochemistry was performed on Calu-6 xenograft tissue. Calu-6 human lung cancer xenografts were established in nude female mice. When the mean tumour volume reached $1.0 \mathrm{~cm}^{3}$, mice were randomised into treatment groups to receive once-daily oral dosing with vandetanib $(50 \mathrm{mg} / \mathrm{kg})$ or vehicle (1\% Tween-80) from 1 to 5 days. On each day of dosing, six animals in each treatment group were sacrificed $4 \mathrm{~h}$ post-dose. All mice received $20 \mu \mathrm{g}$ of VEGF-A (i.v., $30 \mu \mathrm{l}$ volume) $5 \mathrm{~min}$ before sacrifice. Tumours were collected from each animal for IHC analysis. Formalin- and zinc-fixed paraffinembedded tumours (FFPE and ZnFPE, respectively) were sectioned $(4 \mu \mathrm{m})$, dewaxed and rehydrated. IHC was performed on FFPE sections using rabbit monoclonal antibody 55B11 (CST) to detect mouse total VEGFR-2 (16) and rabbit polyclonal antibody CB1764 to detect pY1214 of mouse VEGFR-2 (17). Briefly, antigen retrieval was performed in a pressure cooker (R2100-UK, PickCell Laboratories, Amsterdam, The Netherlands) using S1699 and S3307 buffers (Dako, Ely, UK) for CB1764 and 55B11, respectively. All incubations were carried out at room temperature, and all washes were performed with TBS-T. Sections were blocked using an avidin-biotin kit (X0590, Dako) followed by incubation with 5\% goat serum (Dako) in TBS-T for 20 min. Dilutions of CB1764 (1:50) or $55 \mathrm{~B} 11$ (1:40) in serum block were then added to the sections for $1 \mathrm{~h}$. After washing, a 1:200 dilution of biotinylated goat anti-rabbit immunoglobulin (Dako) was added for $30 \mathrm{~min}$. Following a 30-min incubation in Vectastain ABC-Elite solution (Vector Laboratories, UK), the sections were developed for 10 min in DAB (BioGenex, San Ramon, CA) and counterstained with Carazzi's hematoxylin. ZnFPE sections were used to detect CD31 using rat monoclonal antibody MEC 13.3 (Pharmingen) at a dilution of 1:500. Immunostaining was performed as above except no antigen retrieval was performed, $5 \%$ rabbit serum was employed as block and diluent, and a 1:200 dilution of biotinylated rabbit anti-rat immunoglobulin (Dako) was used. The pVEGFR-2:tVEGFR-2 ratio for each viable xenograft tumour rim was measured as described (17). Briefly, serial sections immunostained for pVEGFR-2 and tVEGFR-2 were scanned into the Automated Cellular Imaging System (ACIS, ChromaVision Medical Systems Inc.). The ACIS MVD (microvessel density) algorithm was employed to generate the mean number of pVEGFR-2- and tVEGFR-2-positive vessel structures per $\mathrm{mm}^{2}$ of viable tissue at the periphery of each tumour and these data were used to calculate the pVEGFR-2: tVEGFR-2 vascular ratio for each viable tumour rim. Analysis of MVD was performed on CD31 immunostained sections of each tumour, using the ACIS and the data expressed as the mean number of CD31-positive vascular structures per $\mathrm{mm}^{2}$ of viable tumour. Statistical analysis was by one- or two-way ANOVA on log-transformed data. Where ANOVA $\mathrm{P}<0.05$, analysis of the vandetanib and vehicle treated groups was subsequently performed using a two-tailed t-test.

Tumour growth studies. Experiments were conducted on 8-12-week-old female SCID mice (CB17/Icr.PrkdcSCID/Crl, Charles River, UK) housed in negative pressure isolators (PFI Systems Ltd, Oxon, UK). PC-9 human tumour cells were maintained in RPMI-1640 media containing 10\% FBS and 1\% L-glutamine at $37^{\circ} \mathrm{C}$ in the presence of $7.5 \% \mathrm{CO}_{2}$. PC-9 human lung tumour xenografts were established as described above for LoVo xenografts. When the mean tumour volume reached $0.3-0.4 \mathrm{~cm}^{3}$, mice were randomized into treatment groups of 10 animals per group to receive once-daily oral dosing with vandetanib (12.5 or $50 \mathrm{mg} / \mathrm{kg}$ ), gefitinib $(12.5 \mathrm{mg} / \mathrm{kg})$, vatalanib (100 mg/kg) or vehicle (1\% Tween-80) for 21 days. Tumour volume was calculated and statistical significance evaluated using a one-tailed t-test. To assess the effects on EGFR phosphorylation and the downstream signalling events in response to acute treatment, a separate set of experiments were performed on 8-12-week-old female SCID mice bearing established PC-9 tumour xenografts. Mice received once-daily oral doses of vandetanib $(50 \mathrm{mg} / \mathrm{kg}, \mathrm{n}=3)$, vatalanib $(100 \mathrm{mg} / \mathrm{kg}, \mathrm{n}=3)$ or vehicle $(1 \%$ Tween-80, $\mathrm{n}=4)$ for 4 days and were sacrificed $2 \mathrm{~h}$ post-dose. Tumours were removed and snap-frozen in liquid nitrogen. Frozen tumours were then homogenized in ice-cold lysis buffer [20 mM Tris, $\mathrm{pH} 7.4 ; 137 \mathrm{mM} \mathrm{NaCl} ; 10 \mathrm{mM}$ EDTA; $1 \%$ Igepal; $2.5 \%$ sodium deoxycholate; protease inhibitors and phosphatase inhibitors 1 and 2 (Sigma, Poole, UK)]. ELISA kits were used according to the manufacturer's 


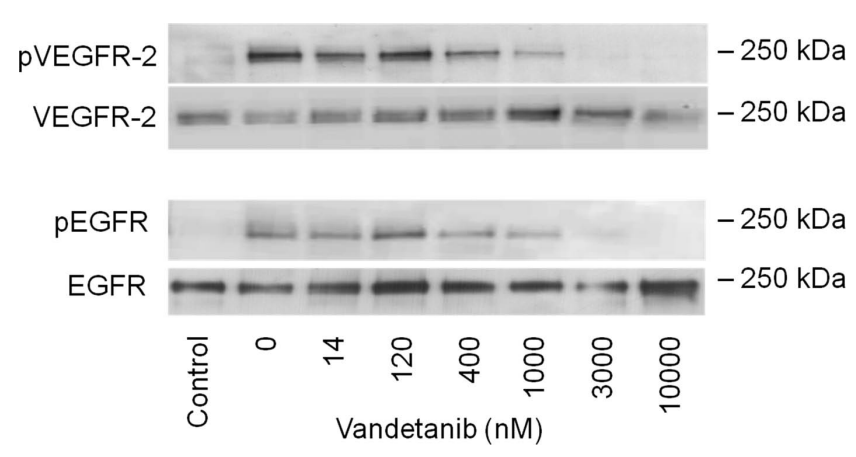

Figure 1. HUVECs were cultured in serum-free medium for $2.5 \mathrm{~h}$ followed by treatment with vandetanib $(0-10,000 \mathrm{nM})$ for $1.5 \mathrm{~h}$, with VEGF-A (50 $\mathrm{ng} / \mathrm{ml})$ or EGF $(1 \mu \mathrm{g} / \mathrm{ml})$ added for the last $5 \mathrm{~min}$ of vandetanib treatment. A representative Western blotting is shown. No VEGF-A or EGF was added to HUVECs in the control lane.

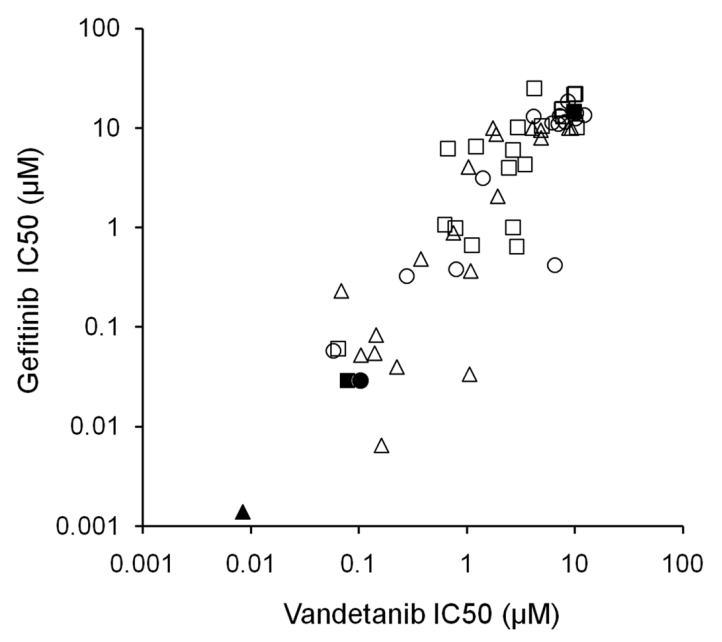

Figure 2. Sensitivity of human tumour cell lines to vandetanib and gefitinib. Human NSCLC ( $\mathrm{n}=22$, squares), colorectal ( $\mathrm{n}=14$, triangles) and SCCHN and esophageal cell lines $(n=20$, circles) were treated with vandetanib of gefitinib for 72-96 h. Mean $\mathrm{IC}_{50}$ values for the growth inhibitory effects of vandetanib and gefitinib are plotted for each cell line. The presence of activating mutations in EGFR is indicated (filled symbols) and comprised NSCLC (PC-9, H1975); colorectal (SW48) and esophageal (KYSE-450) cell lines.

instructions to measure pEGFR (DYC1905, R\&D Systems) and M30 (P10010, DiaPharma, West Chester, OH). Statistical analysis was by one-way ANOVA and a two-tailed t-test.

\section{Results}

Inhibition of pVEGFR-2 and pEGFR in HUVECs. Vandetanib, in a concentration-related manner, inhibited ligand-stimulated phosphorylation of both VEGFR-2 and EGFR in HUVECs (Fig. 1); $\mathrm{IC}_{50}$ values (mean $\pm \mathrm{SE} ; \mathrm{n}=4$ independent experiments) were $0.4 \pm 0.13 \mu \mathrm{M}$ (pVEGFR-2) and $1.1 \pm 0.47 \mu \mathrm{M}$ (pEGFR).

Inhibition of tumour cell growth. Using a viable cell number end-point, the sensitivity of tumour cell lines to cell growth inhibition by vandetanib was significantly correlated with sensitivity to gefitinib (NSCLC panel, $\mathrm{n}=22, \mathrm{r}=0.77, \mathrm{P}<0.0001$; colorectal panel, $\mathrm{n}=14, \mathrm{r}=0.79, \mathrm{P}=0.0007$; $\mathrm{SCCHN} / \mathrm{esophageal}$ panel, $n=20, r=0.80, P<0.0001$ ) (Fig. 2). Of the four cell lines
$\underline{P C-9}$
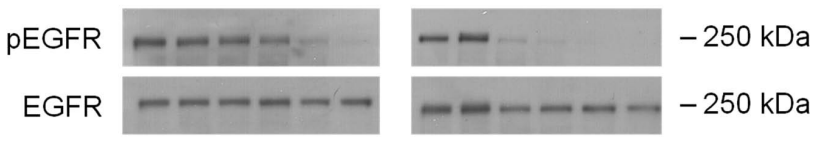

$\underline{\mathrm{H} 1975}$

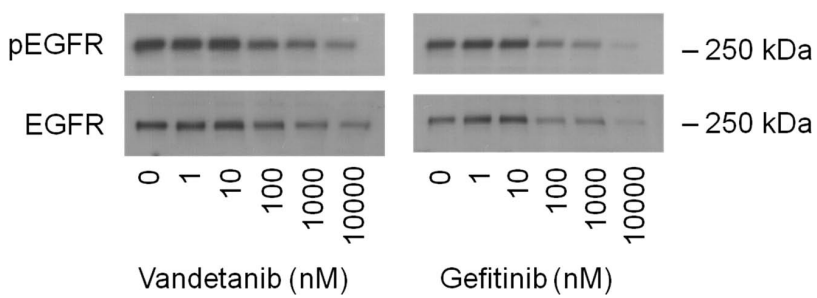

Figure 3. Inhibition of pEGFR in PC-9 and H1975 human NSCLC cells by vandetanib and gefitinib. PC-9 (upper panel) and H1975 (lower panel) cells were seeded into medium containing $10 \%$ FCS and then treated with vandetanib or gefitinib $(0-10 \mu \mathrm{M})$ for $4 \mathrm{~h}$. Cell lysates were prepared for Western blot analysis of levels of phosphorylated and total VEGFR-2 and EGFR. Representative blots are shown.

with known activating mutations in the EGFR gene, three (KYSE-450, PC-9, SW48) (18-20) were among the most sensitive to growth inhibition by both vandetanib and gefitinib, whereas one (H1975) (21), with an activating mutation and a T790M 'gatekeeper' mutation, was among the most resistant (Fig. 2).

Inhibition of pEGFR in human NSCLC cells. Treatment with vandetanib or gefitinib inhibited pEGFR in PC-9 and H1975 cells cultured under basal growth conditions (Fig. 3). In PC-9 cells, the $\mathrm{IC}_{50}$ values for the inhibition of pEGFR by ELISA were $0.39 \pm 0.09 \mu \mathrm{M}$ (vandetanib) and $0.0029 \pm 0.0007 \mu \mathrm{M}$ (gefitinib). In H1975 cells, the mean $\mathrm{IC}_{50}$ value for both vandetanib and gefitinib was $>10 \mu \mathrm{M}$. Data were calculated from the mean $\pm \mathrm{SE}$ of six experiments (two independent assays run in triplicate).

Phenotypic effects in human tumour cells. Compared with controls, vandetanib, in a concentration-related manner, increased both the proportion of cells in G1 (Fig. 4A) and the proportion of cells undergoing apoptosis (Fig. 4B). These effects were most marked in cells lines that were more sensitive to vandetanib in the MTS assays (PC-9 and OE21 cell lines). Similar phenotypic effects were seen with gefitinib (data not shown).

Lung and tumour pharmacodynamic assays. Treating nude mice bearing established LoVo human colon cancer xenografts with vandetanib ( 25 or $100 \mathrm{mg} / \mathrm{kg} / \mathrm{day}$, for $1-4$ days) had significant effects on pVEGFR-2 levels in lung, and pEGFR levels in lung and tumour tissue (Fig. 5). Using two-way ANOVA with treatment group and number of doses as fixed effects, the effect of treatment group was statistically significant $(\mathrm{P}<0.01)$ on levels of pVEGFR-2 and pEGFR in lung, as well as pEGFR in tumour tissue. In the lung, compared with control, treatment with vandetanib (25 or $100 \mathrm{mg} / \mathrm{kg}$ ) reduced pVEGFR-2 levels by $70.7 \%$ (95\% CI 46.9\%, 83.8\%; P=0004) and $76.9 \%$ (95\% CI 58.2\%, 87.2\%; P<0001), respectively, and pEGFR 


\section{A}
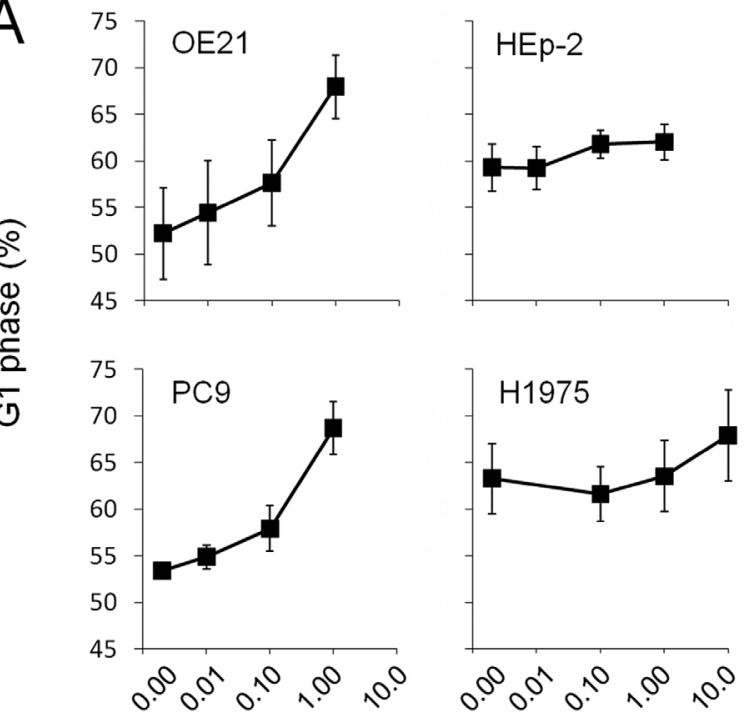

Vandetanib $(\mu \mathrm{M})$
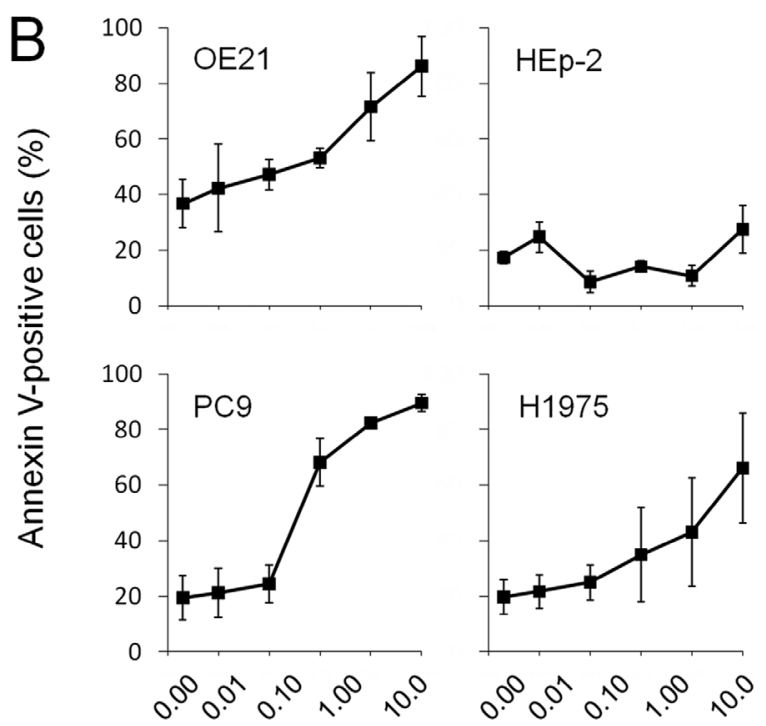

Vandetanib $(\mu \mathrm{M})$

Figure 4. Phenotypic effects of vandetanib in human tumour cells. NSCLC (PC-9, H1975), esophageal (OE21) and SCCHN (HEp-2) cells were treated with vandetanib $(0-10 \mu \mathrm{M})$ for 24-72 $\mathrm{h}$. Cell were stained for DNA content using propidium iodide and the proportion (\%) of cells in G1 phase of the cell cycle determined by FACS (A, mean \pm SEM, $n=6$ experiments). Alternatively, vandetanib treated cells were stained for annexin $\mathrm{V}$ to determine extent (\%) of apoptosis (B, mean \pm SEM, $n=4$ experiments).

levels by $56.7 \%$ (95\% CI: $25.7 \%, 74.8 \% ; \mathrm{P}=0.007)$ and $48.3 \%$ (95\% CI: $11.2 \%, 69.9 \%$; $\mathrm{P}=0.04)$, respectively. In tumour tissue, vandetanib treatment $(25$ or $100 \mathrm{mg} / \mathrm{kg}$ ) reduced pEGFR levels by $56.7 \%$ (95\% CI: $38.1 \%, 69.7 \% ; \mathrm{P}<0.0001)$ and $58.4 \%(95 \%$ CI: $40.6 \%, 70.9 \%$; $\mathrm{P}<0.0001)$. In contrast to treatment group, there was no significant effect of number of doses on pVEGFR-2 or pEGFR levels in the lung. There was an effect of the number of doses on tumour pEGFR levels though this was not clearly relate to dosing with vandetanib and may more reflect day to day variation in tumour handling and processing (data

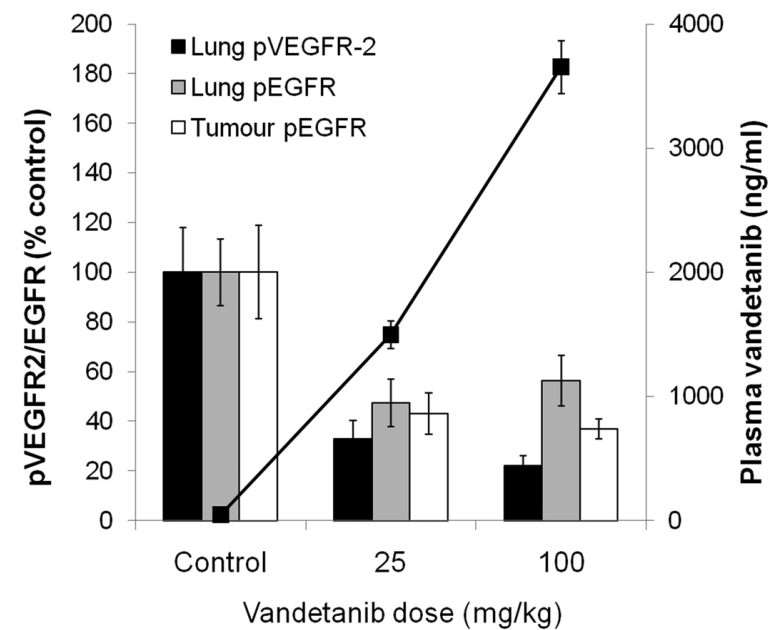

Figure 5. Western blot analysis of pVEGFR-2 and pEGFR levels in lung and tumour tissue. Three groups of nude mice ( $\mathrm{n}=12$ per group) bearing established $\left(0.5 \mathrm{~cm}^{3}\right)$ LoVo human colorectal cancer subcutaneous xenografts were randomised to receive vandetanib ( $25 \mathrm{or} 100 \mathrm{mg} / \mathrm{kg}$ ) or drug vehicle once daily for 1-4 days. Mean ( \pm SEM) levels of lung pVEGFR-2 (black columns), lung pEGFR (grey columns) and LoVo tumour pEGFR (white columns) are shown for vandetanib treated animals relative to vehicle-treated controls. Mean ( \pm SEM) plasma concentrations of vandetanib (black squares) are also shown.

not shown). The pharmacological effects of $25 \mathrm{mg} / \mathrm{kg} /$ day vandetanib (mean plasma drug concentration $1497 \pm 109 \mathrm{ng} / \mathrm{ml}$ ) were considered the near maximum achievable in this model since increasing the vandetanib dose to $100 \mathrm{mg} / \mathrm{kg} / \mathrm{day}$ substantially increased mean plasma drug concentration $(3653 \pm 211 \mathrm{ng} / \mathrm{ml})$ without further reducing levels of pVEGFR-2 or pEGFR in lung or tumour tissue (Fig. 5).

Immunohistochemical analysis of pVEGFR-2. Treatment with vandetanib $(50 \mathrm{mg} / \mathrm{kg})$ caused a small $(25 \%)$ but significant reduction in tumour microvessel density (MVD, $\mathrm{P}<0.004$, ANOVA) in the Calu-6 human lung cancer xenograft model as determined by CD31 staining (Fig. 6A). Although there was no statistically significant effect of number of doses on MVD, the data did suggest that greater differences (up to $46 \%$ reduction in MVD) between vehicle and vandetanib treated groups after more prolonged dosing (Fig. 6A). In contrast, vandetanib treatment produced a marked $(75 \%, \mathrm{P}<0.0001)$ reduction in pVEGFR-2 expression (Fig. 6B) that was apparent even after the first treatment dose. In these experiments, both pVEGFR-2 and total VEGFR-2 staining was restricted to blood vessels, with no evidence of staining of tumour cells or non-endothelial stromal tissue. Vandetanib treatment had no significant effect on the number of microvessels expressing total VEGFR-2, or the intensity of total VEGFR-2 staining (data not shown). Since a reduction in CD31 MVD was not apparent until after the third dose of vandetanib, the acute reduction in pVEGFR-2 on day 1 after dosing suggested a potential delayed phenotypic response to VEGFR-2 signalling inhibition in this tumour model.

Effects on established human lung tumour xenografts. In a PC-9 lung tumour xenograft model, harbouring an activating EGFR mutation (delE746-A570), once-daily oral administration 


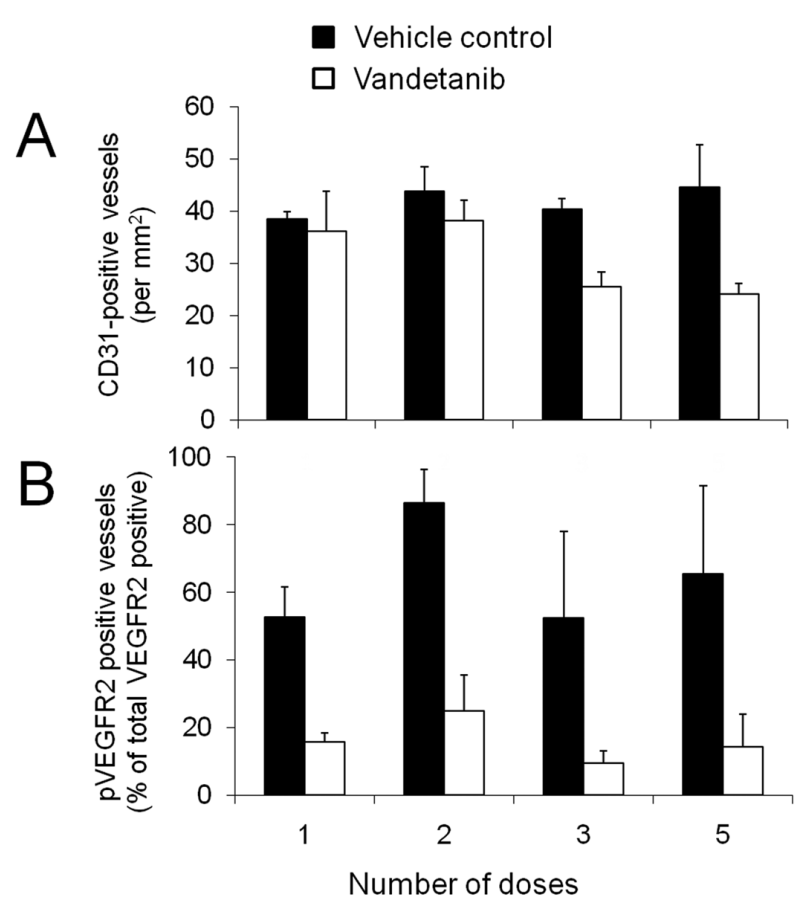

Figure 6. Immunohistochemical analysis of pVEGFR levels in Calu-6 tumour tissue. Two groups of nude mice ( $n=24$ per group) bearing established $\left(0.5-1.0 \mathrm{~cm}^{3}\right)$ Calu-6 human lung cancer subcutaneous xenografts were randomised to receive vandetanib $(50 \mathrm{mg} / \mathrm{kg})$ or drug vehicle daily for $1-5$ days. Cohorts of animals from each group $(n=6$ per cohort) were sacrificed $4 \mathrm{~h}$ after dosing on days 1, 2, 3 or 5. Tumours were assessed by IHC for expression of CD31, total VEGFR-2, and pVEGFR-2.(A) Tumour microvessel density (mean \pm SEM, $n=4$ animals per treatment group at each time-point) measured by CD31 staining in vehicle-treated (black columns) and vandetanibtreated (white columns). (B) Expression of pVEGFR-2 relative to expression of total VEGFR-2 (mean percentage \pm SEM, $n=3-5$ animals per treatment group at each time-point).

of vandetanib (12.5 or $50 \mathrm{mg} / \mathrm{kg})$ or gefitinib $(12.5 \mathrm{mg} / \mathrm{kg})$ for 21 days significantly reduced tumour growth compared with vehicle treated controls $(\mathrm{P}<0.001)$, producing marked tumour regressions within 7-14 days of the onset of dosing. At the higher dose of vandetanib, tumours did not show evidence of re-growth when treatment was stopped (Fig. 7A). In contrast, once-daily oral administration of vatalanib $(100 \mathrm{mg} / \mathrm{kg})$, a selective VEGFR tyrosine kinase inhibitor, did not induce tumour regression and had only modest effects on tumour growth which did not reach statistical significance after 21 days of dosing $(\mathrm{P}=0.06)$.

In PC-9 tumours from mice treated for 4 days with vandetanib $(50 \mathrm{mg} / \mathrm{kg})$, levels of pEGFR were $84 \%$ lower $(\mathrm{P}<0.001)$ and levels of M30 (a marker of early apoptosis events) were 3 -fold higher $(\mathrm{P}<0.001)$ than in tumours from vehicle treated controls (Fig. 7B and C). In contrast, in tumours from animals treated with vatalanib $(100 \mathrm{mg} / \mathrm{kg})$, levels of pEGFR and M30 were not significantly different from controls (Fig. 7B and C).

\section{Discussion}

Vandetanib was previously identified as an orally available small molecule inhibitor of VEGF-dependent angiogenesis, with the additional potential of inhibiting EGFR-dependent cell growth (1). Both of these signalling pathways are validated targets for cancer therapy in NSCLC $(22,23)$. Although vandetanib
A
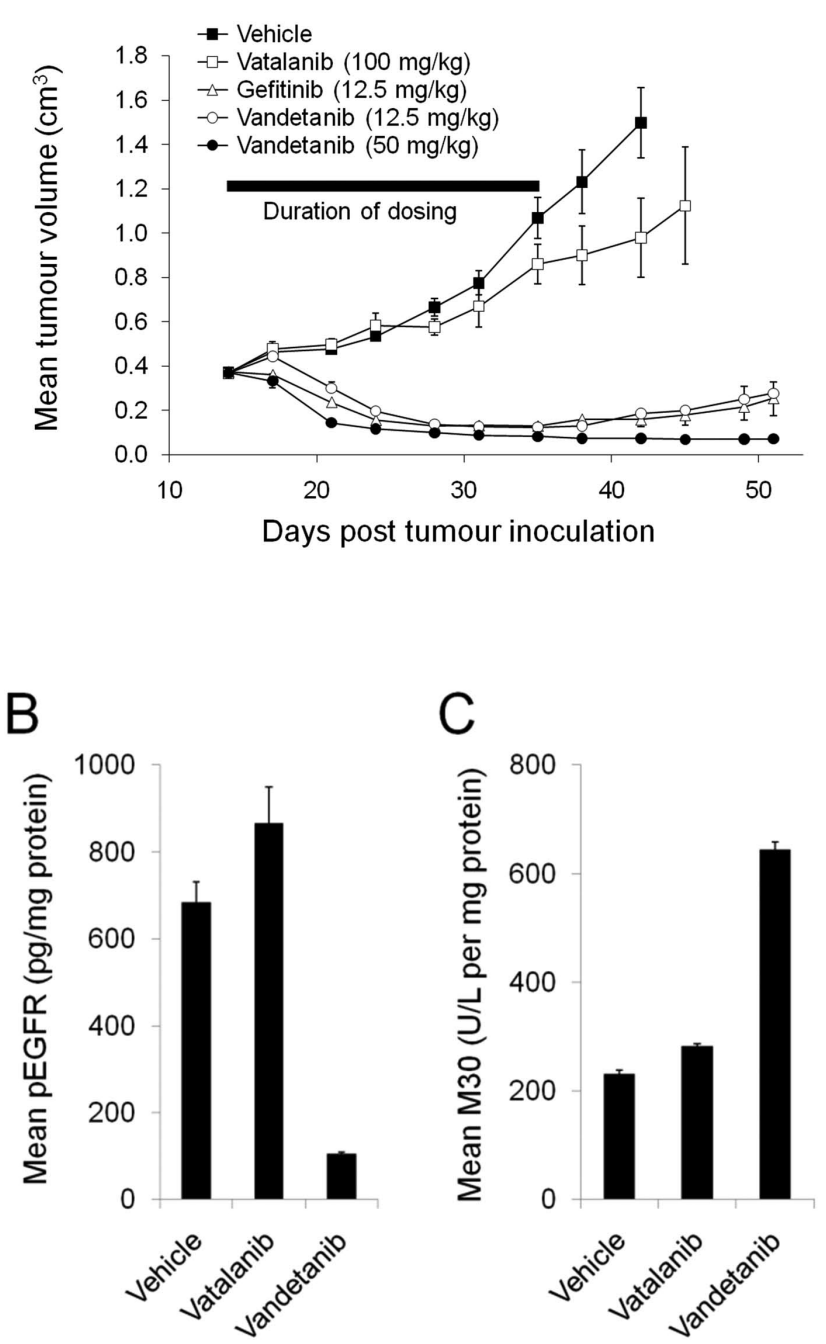

Figure 7. Effects of vandetanib treatment on established PC-9 xenografts. (A) SCID mice bearing established PC-9 human NSCLC xenografts were randomised to receive vatalanib (100 mg/kg/day), gefitinib $(12.5 \mathrm{mg} / \mathrm{kg} /$ day $)$, vandetanib $(12.5 \mathrm{mg} / \mathrm{kg} / \mathrm{day} ; 50 \mathrm{mg} / \mathrm{kg} / \mathrm{day})$ or drug vehicle alone by oral gavage ( $\mathrm{n}=10$ animals per group). Animals were dosed for 21 days. Tumour volume was recorded during drug dosing and for up to 16 days after treatment was stopped. (B and C) SCID mice bearing established PC-9 human NSCLC xenografts were randomised to receive vatalanib $(100 \mathrm{mg} /$ $\mathrm{kg} /$ day, $\mathrm{n}=3)$, vandetanib $(50 \mathrm{mg} / \mathrm{kg} / \mathrm{day}, \mathrm{n}=3)$ or drug vehicle alone $(\mathrm{n}=4)$ by oral gavage. Two hours after the fourth dose, animals were sacrificed and the tumours collected and assayed for pEGFR (B) and M30 (C) protein levels. Values are mean \pm SEM.

combined with docetaxel has demonstrated significant prolongation of progression free survival compared with docetaxel alone, and vandetanib monotherapy has demonstrated equivalent efficacy to erlotinib in phase III studies (24; Natale et al, J Clin Oncol 27 (Suppl): abs. 8009, 2009), the mechanisms underlying this clinical activity are not known.

In the present work, there was an approximately 3-fold difference in $\mathrm{IC}_{50}$ values for inhibition of pVEGFR-2 compared with pEGFR in HUVECs in vitro which is in line with a previous report for relative inhibition of VEGF- versus EGFstimulated HUVEC proliferation. Taken together, these data suggest that VEGFR signalling may be more potently inhibited by vandetanib than EGFR signalling in HUVECs, although 
the $\mathrm{IC}_{50}$ data suggest that both signalling pathways have the potential to be inhibited in endothelial cells in man.

In tumour cell growth inhibition assays, the effects of vandetanib were highly correlated with those of gefitinib, including phenotypic effects (cell cycle, apoptosis) in both sensitive and resistant cell lines. An EGFR T790M mutation was associated with resistance to the effects of both vandetanib and gefitinib (pEGFR, cell proliferation, cell cycle, apoptosis). The data suggest that the direct anti-tumour effects of vandetanib are primarily due to inhibition of EGFR in these tumour cell lines, and these effects may be observed for some cell lines at vandetanib concentrations well below the plasma drug levels achieved in the clinic. Therefore, in the clinic, there is the potential for substantial direct effects against tumour cells highly dependent on EGFR signalling, for example those with activating EGFR mutations. Although some cell lines are highly resistant to direct anti-tumour effects of vandetanib, this could be ameliorated in the clinical situation where indirect anti-tumour activity could result from inhibition of VEGFR and EGFR signalling in endothelial cells.

Two hours after treatment of mice with a single dose of vandetanib at $25 \mathrm{mg} / \mathrm{kg}$ plasma drug levels were similar to those reached at steady-state after dosing at $300 \mathrm{mg} /$ day in man. Using a Western blot assay, this dose of vandetanib was shown to significantly inhibit both pVEGFR-2 and pEGFR in normal tissue (lung), and pEGFR in tumour in vivo. Interestingly, substantially increasing the plasma drug concentration by dosing vandetanib at $100 \mathrm{mg} / \mathrm{kg}$ did not further inhibit pVEGFR-2 or pEGFR, suggesting that $25 \mathrm{mg} / \mathrm{kg}$ was at or above the top of the dose-response curve for this assay, although lower doses were not tested.

It was not possible to accurately assess tumour pVEGFR-2 by quantitative Western blotting as the low proportion of endothelial cells within tumour tissue makes this assay too insensitive. However, using quantitative immunohistochemistry, the extent of pVEGFR-2 inhibition by vandetanib in normal lung endothelium and tumour endothelium was shown to be broadly similar. Therefore, these in vivo data suggested that vandetanib inhibits both pVEGFR-2 and pEGFR similarly in both normal and tumour tissue with no evidence for differential sensitivity of signalling inhibition in tumours.

Previous in vivo data showed that vandetanib has broad antitumour activity, including activity in xenografts models that are insensitive to selective EGFR TKIs, supporting the concept that the key biological target in these models was VEGFR-2 signalling in the endothelial cells of the tumour stroma. Thus, inhibition of VEGF-dependent angiogenesis indirectly inhibited tumour growth, producing growth suppression, or tumour stasis during prolonged vandetanib dosing. However, in vitro data in the present study suggested that some tumour cells, including those with activating EGFR mutations, are particularly sensitive to direct antitumour effects of vandetanib. In the PC-9 xenograft model, the in vivo anti-tumour effects of vandetanib were very similar to those of gefitinib as has been reported by other workers $(25,26)$, producing rapid tumour regressions associated with induction of tumour cell apoptosis. Interestingly, at a higher dose, there was no evidence of tumour regrowth in a 2 -week time period after which treatment was stopped. Although further studies are needed, this suggests, that continuous chronic therapy with vandetanib may not always be required in some highly responsive tumours. The marked anti-tumour activity of vandetanib in the PC-9 xenograft model is in contrast to the more modest growth suppression produced by vatalanib, a highly selective VEGFR signalling inhibitor, and to the more modest activity of vandetanib in xenograft models insensitive to EGFR TKIs (1,27).

In conclusion, our preclinical data demonstrate that vandetanib inhibits both VEGFR-2 and EGFR signalling at clinically relevant drug levels in preclinical models of human cancer. In addition, our data suggest that where tumour cells are highly sensitive to growth inhibition by vandetanib then direct antitumour effects (e.g., through EGFR inhibition) will dominate, whereas for tumour cells refractory to growth inhibition by vandetanib, then indirect antitumour effects (e.g., through VEGFR-2 inhibition) will dominate. However, a large proportion of tumour cells are likely to have an intermediate level of sensitivity to growth inhibition by vandetanib, and in these cases, both direct and indirect effects are likely to significantly contribute to the observed antitumour effects. Biomarkers of direct effects (e.g., EGFR gene mutations) may be most useful in identifying patients with potential for greatest benefit, by predicting durable tumour shrinkage and increased tumour response rate. In contrast, biomarkers of benefit from indirect inhibition of tumour growth may be most useful in those patients whose tumours are insensitive to the direct effects of vandetanib, where the clinical benefit may be reflected primarily by tumour growth delay and increased progression-free survival (28).

\section{References}

1. Wedge SR, Ogilvie DJ, Dukes M, et al: ZD6474 inhibits vascular endothelial growth factor signaling, angiogenesis, and tumor growth following oral administration. Cancer Res 62: 4645-4655, 2002.

2. Ryan AJ and Wedge SR: ZD6474 - a novel inhibitor of VEGFR and EGFR tyrosine kinase activity. Br J Cancer 92 (Suppl. 1): S6-S13, 2005.

3. Wakeling AE, Guy SP, Woodburn JR, et al: ZD1839 (Iressa): an orally active inhibitor of epidermal growth factor signaling with potential for cancer therapy. Cancer Res 62: 5749-5754, 2002

4. Heymach JV, Johnson BE, Prager D, et al: Randomized, placebocontrolled phase II study of vandetanib plus docetaxel in previously treated non small-cell lung cancer. J Clin Oncol 25: 4270-4277, 2007.

5. Natale RB, Bodkin D, Govindan R, et al: Vandetanib versus gefitinib in patients with advanced non-small-cell lung cancer: results from a two-part, double-blind, randomized phase II study. J Clin Oncol 27: 2523-2529, 2009.

6. Heymach JV, Paz-Ares L, De Braud F, et al: Randomized phase II study of vandetanib alone or with paclitaxel and carboplatin as first-line treatment for advanced non-small-cell lung cancer. J Clin Oncol 26: 5407-5415, 2008.

7. Morabito A, Piccirillo MC, Falasconi F, et al: Vandetanib (ZD6474), a dual inhibitor of vascular endothelial growth factor receptor (VEGFR) and epidermal growth factor receptor (EGFR) tyrosine kinases: current status and future directions. Oncologist 14: 378-390, 2009.

8. Kiura K, Nakagawa K, Shinkai T, et al: A randomized, doubleblind, phase IIa dose-finding study of Vandetanib (ZD6474) in Japanese patients with non-small cell lung cancer. J Thorac Oncol 3: 386-393, 2008.

9. Ciardiello $\mathrm{F}$ and Tortora G: EGFR antagonists in cancer treatment. N Engl J Med 358: 1160-1174, 2008.

10. Jain RK, Duda DG, Willett CG, et al: Biomarkers of response and resistance to antiangiogenic therapy. Nat Rev Clin Oncol 6: 327-338, 2009. 
11. Annunziata CM, Walker AJ, Minasian L, et al: Vandetanib designed to inhibit VEGFR2 and EGFR signaling, had no clinical activity as monotherapy for recurrent ovarian cancer and no detectable modulation of VEGFR2. Clin Cancer Res 16: 664-672, 2010.

12. Baselga J, Trigc JM, Rojo F, et al: Pharmacodynamic assessment of ZD6474 (ZACTIMA (TM)) in the skin of patients with previously treated metastatic breast cancer. Clin Cancer Res 11: S9027-S9027, 2005.

13. Barker AJ, Gibson KH, Grundy W, et al: Studies leading to the identification of ZD1839 (IRESSA): an orally active, selective epidermal growth factor receptor tyrosine kinase inhibitor targeted to the treatment of cancer. Bioorg Med Chem Lett 11: 1911-1914, 2001

14. Hennequin LF, Stokes ES, Thomas AP, et al: Novel 4-anilinoquinazolines with $\mathrm{C}-7$ basic side chains: design and structure activity relationship of a series of potent, orally active, VEGF receptor tyrosine kinase inhibitors. J Med Chem 45: 1300-1312, 2002.

15. Wedge SR, Kendrew J, Hennequin LF, et al: AZD2171: a highly potent, orally bioavailable, vascular endothelial growth factor receptor-2 tyrosine kinase inhibitor for the treatment of cancer. Cancer Res 65: 4389-4400, 2005.

16. Smith NR, Baker D, James NH, et al: Vascular endothelial growth factor receptors VEGFR-2 and VEGFR-3 are localized primarily to the vasculature in human primary solid cancers. Clin Cancer Res 16: 3548-3561, 2010.

17. Smith NR, James NH, Oakley I, et al: Acute pharmacodynamic and antivascular effects of the vascular endothelial growth factor signaling inhibitor AZD2171 in Calu-6 human lung tumor xenografts. Mol Cancer Ther 6: 2198-2208, 2007.

18. Guo M, Liu S, Herman JG, Zhuang H and Lu F: Gefitinibsensitizing mutation in esophageal carcinoma cell line Kyse450. Cancer Biol Ther 5: 152-155, 2006.

19. Arao T, Fukumoto H, Takeda M, Tamura T, Saijo N and Nishio K: Small in-frame deletion in the epidermal growth factor receptor as a target for ZD6474. Cancer Res 64: 9101-9104, 2004.
20. Yeh JJ, Routh ED, Rubinas T, et al: KRAS/BRAF mutation status and ERK1/2 activation as biomarkers for MEK1/2 inhibitor therapy in colorectal cancer. Mol Cancer Ther 8: 834-843, 2009.

21. Pao W, Miller VA, Politi KA, et al: Acquired resistance of lung adenocarcinomas to gefitinib or erlotinib is associated with a second mutation in the EGFR kinase domain. PLoS Med 2: e73, 2005.

22. Shepherd FA, Rodrigues Pereira J, Ciuleanu T, et al: Erlotinib in previously treated non-small-cell lung cancer. N Engl J Med 353: 123-132, 2005.

23. Sandler A, Gray R, Perry MC, et al: Paclitaxel-carboplatin alone or with bevacizumab for non-small-cell lung cancer. N Engl J Med 355: 2542-2550, 2006.

24. Herbst RS, Sun Y, Eberhardt WE, et al: Vandetanib plus docetaxel versus docetaxel as second-line treatment for patients with advanced non-small-cell lung cancer (ZODIAC): a double-blind, randomised, phase 3 trial. Lancet Oncol 11: 619-626, 2010.

25. Naumov GN, Nilsson MB, Cascone T, et al: Combined vascular endothelial growth factor receptor and epidermal growth factor receptor (EGFR) blockade inhibits tumor growth in xenograft models of EGFR inhibitor resistance. Clin Cancer Res 15: 3484-3494, 2009.

26 Taguchi F, Koh Y, Koizumi F, Tamura T, Saijo N and Nishio K: Anticancer effects of ZD6474, a VEGF receptor tyrosine kinase inhibitor, in gefitinib (Iressa)-sensitive and resistant xenograft models. Cancer Sci 95: 984-989, 2004.

27. Ichihara $\mathrm{E}$, Ohashi K, Takigawa N, et al: Effects of vandetanib on lung adenocarcinoma cells harboring epidermal growth factor receptor T790M mutation in vivo. Cancer Res 69: 5091-5098, 2009.

28. Conrad C, Ischenko I, Kohl G, et al: Antiangiogenic and antitumor activity of a novel vascular endothelial growth factor receptor-2 tyrosine kinase inhibitor ZD6474 in a metastatic human pancreatic tumor model. Anticancer Drugs 18: 569-579, 2007. 